\title{
Enhanced acoustical transmission and beaming effect through a single aperture
}

\author{
J. Christensen, ${ }^{1, *}$ L. Martín-Moreno, ${ }^{2}$ and F. J. García-Vidal ${ }^{1}$ \\ ${ }^{1}$ Departamento de Física Teórica de la Materia Condensada, Universidad Autónoma de Madrid, E-28049 Madrid, Spain \\ ${ }^{2}$ Instituto de Ciencia de Materiales de Aragón (ICMA) and Departamento de Física de la Materia Condensada, \\ CSIC-Universidad de Zaragoza, E-50009 Zaragoza, Spain \\ (Received 15 February 2010; revised manuscript received 26 March 2010; published 4 May 2010)
}

\begin{abstract}
In this paper we explore from a fundamental theoretical point of view, transmission phenomena of acoustic waves transferred through a single subwavelength slit milled into a sound-hard plate that is textured by surface corrugations. It is shown that the enhanced acoustical transmission unambiguously is linked to the excitation of acoustic surface waves and Fabry-Perot modes within the aperture. With the former resonant condition, we give a prescription on how these surface waves are induced and connected to the formation of a collimated sound beam in the far field.
\end{abstract}

DOI: 10.1103/PhysRevB.81.174104

PACS number(s): 78.66.Bz, 42.25.Bs, 41.20.Jb, 73.20.Mf

\section{INTRODUCTION}

It is now more than one decade ago since Ebbesen et al. ${ }^{1}$ discovered the extraordinary optical transmission (EOT) of light through an array of subwavelength holes in a metallic film. Since then, this discovery has entailed numerous publications in terms of fundamental research but also regarding technological aspects. To this we can count the ability to enhance light transmission through a single aperture and to compress the diffracted radiation into a narrow beam. ${ }^{2} \mathrm{Al}-$ though there has been a great deal of debate and controversy behind the mechanism of EOT, it is now widely accepted that a resonant excitation of surface-plasmon polaritons (SPPs) on and through the structure gives rise to the observation of enhanced transmission. In particular, the discovery of EOT spectrally below the optical regime, at megahertz and tetrahertz frequencies, where bound surface modes in the form of plasmons only are located weak, led to the consideration of the sustainability of spoof SPPs in perfect conducting materials. ${ }^{3,4}$ In the case of sound waves several work on both theory and experiments has been performed in order to study the acoustical analogy. ${ }^{5-12}$ One of the main difference between holey structures for light and sound propagation is the absence of polarization for a longitudinal-acoustic wave, which always makes the lowest waveguide mode inside apertures propagative, exhibiting no cutoff. ${ }^{8}$ Also nonopaque screens made out of sound-soft materials, which is giving rise to the coupling with structure-borne sound, make a clear statement on not to be a trivial analogy to its optical counterpart. ${ }^{11}$

In this paper we want to call the attention to the theoretical study on the enhanced acoustical transmission and beaming effect through a single slit in a perfect rigid body (PRB). Due to a large impedance contrast, this PRB approximation is well suited for fluid-borne sound funneled through rigid materials such as steel or brass. This paper is a follow up to a Brief Communication where we presented simulations regarding sound transmission and collimation of an isolated aperture flanked by corrugations, on a superior level. ${ }^{7}$ We provide detailed derivations on an exact formalism which enables an entire thorough understanding of the problem given. As we are introducing corrugations into the rigid structure, we are able to study the scattering properties and its influence on the sound transmission such as the formation of the compressed beam. The basic mechanisms presented here are evocative of those found for electromagnetic radiation. ${ }^{2,13,14}$ Also, the current methodology applied adverts to the one used for the simulation of other finite plasmonic systems. ${ }^{15,16}$ Similar to SPPs, we demonstrate how the right tuning of acoustic surface waves (ASWs) together with Fabry-Perot (FP) such as groove-cavity resonances is giving rise to enhanced performances of sound on a subwavelength scale.

\section{THEORETICAL FORMALISM}

Linear acoustics comprises small pressure fluctuation that forms a traveling wave of low intensity. Consider an inviscid (lossless) fluid (liquid or gas) that is at rest. To connect the motion of the fluid with its compression or expansion, a relationship between the particle velocity $\boldsymbol{v}$ and the mass density $\rho$ is to be deduced. Regarding the forces, one has to apply Newton's second law and relating the sum of the forces acting on an element of fluid to its acceleration or rate of change in momentum. These two constituents are the foundations to describe acoustic waves of small disturbances in the absence of viscosity (constant entropy), that are gathered within the linearized Euler's equations,

$$
\frac{\partial \rho}{\partial t}+\boldsymbol{\nabla} \cdot(\rho \boldsymbol{v})=0, \quad \frac{\partial v}{\partial t}+(\boldsymbol{v} \cdot \boldsymbol{\nabla}) \boldsymbol{v}=-\frac{\boldsymbol{\nabla} p}{\rho} .
$$

In Eq. (1), energy terms are disregarded and will not be part due to the absence of thermal gradients. For the pressure $p$, velocity $\boldsymbol{v}$, and density $\rho$, one can write: $p=p_{0}+p^{\prime}, \boldsymbol{v}=\boldsymbol{v}_{0}$ $+\boldsymbol{v}^{\prime}$, and $\rho=\rho_{0}+\rho^{\prime}$, where the terms $p_{0}, \boldsymbol{v}_{0}$, and $\rho_{0}$ denote the background pressure, velocity $\left(\boldsymbol{v}_{0}=0\right)$, and density in an undisturbed medium, respectively. The primed quantities $p^{\prime}$, $\boldsymbol{v}^{\prime}$, and $\rho^{\prime}$ describe the variation in the corresponding magnitudes due to the presence of a low-amplitude acoustic field in the medium. If one now substitutes those quantities into Eq. (1) and only linear terms in the primed quantities are taken into account while all higher-order terms are neglected, one obtains two simple equations, 


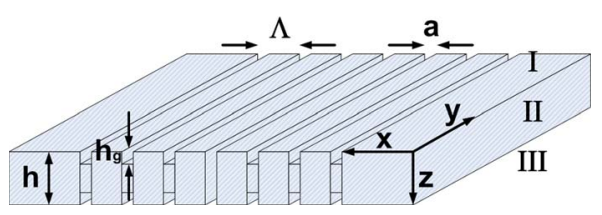

FIG. 1. (Color online) Schematics of a single slit surrounded by finite corrugations, made out of a perfect rigid body, impenetrable for airborne sound waves impinging on the structure.

$$
\boldsymbol{\nabla} \boldsymbol{v}^{\prime}-\frac{i \omega}{c_{0}^{2} \rho_{0}} p^{\prime}=0, \quad \boldsymbol{\nabla} p^{\prime}-i \omega \rho_{0} \boldsymbol{v}^{\prime}=0
$$

that after straightforward algebra yield a simple wave equation for linear sound propagation in fluids. Here, use has been made of monochromatic radiation and the isentropic relation $p^{\prime}=\left(\frac{\partial p}{\partial \rho_{0}}\right)_{s} \rho^{\prime}=c^{2} \rho^{\prime}$ upon assuming adiabatic and reversible conditions. For more details regarding this derivation one should refer to Refs. 17 and 18. Figure 1 is the structure under the present examination devoted to the study of collimation and enhanced transmission of sound waves. This single isolated slit (width $a$ ) milled into a sound-hard plate (thickness $h$ ) is textured by indentations (height $h_{g}$ ) at the upper and the lower interface (separation $\Lambda$ ). In order to study the sound phenomena theoretically, we will start out defining a supercell with lattice parameter $L_{x}$ along the $x$ axis. Later, as we are going to study the enhanced transmission of sound through a single slit flanked by a finite number of surrounding corrugations, the limit $L_{x} \rightarrow \infty$ must be taken. We define an index of refraction for sound as $n=c_{0} / c_{p}$ which is nothing but $\sqrt{K_{0} / \rho_{0}} \sqrt{\rho_{p} / K_{p}}$, with $c, K$, and $\rho$ representing the phase velocity (thermodynamic speed of sound), the bulk modulus, and density, respectively, where the zero and $p$ indices refer to a reference medium (such as air) and phase (any arbitrary medium), respectively. Consequently it is convenient to rewrite $n=\sqrt{\rho_{r} / K_{r}}$ into relative terms, which gives a unity refraction index for air. Initially we will assume the structure given in Fig. 1 to be periodic of constant $L_{x}$ and starting to deduce a wave description in region $I$. In this particular periodic case, we expand the wave into Bloch states by means of a reciprocal-lattice vector. Note, as of translational invariance along the $y$ axis of the structure, it is sufficient to regard the sagittal $(x z)$ plane. Also, only the $z$-component $v_{z}^{\prime}$ of the velocity vector will be taking into account because $v_{z}^{\prime}$ is employed for the matching technique and in order to simplify the notations, we choose to reject the primes in $p^{\prime}$ and $v_{z}^{\prime}$ as given in Eq. (2), though it is clear that acoustical quantities are considered. The acoustic field associated to the incident and the resulting reflected wave, $(z<0)$, represented as a sum of plane waves weighted with their corresponding reflection coefficients $R_{\gamma}$, is

$$
\begin{gathered}
\left|p^{I}(z)\right\rangle=Y_{k_{z}^{0}}^{I}\left|k_{x}^{0}\right\rangle e^{i k_{z}^{0} z}+\sum_{\gamma=-\infty}^{\infty} R_{\gamma} Y_{k_{z}^{\gamma}}^{I}\left|k_{x}^{\gamma}\right\rangle e^{-i k_{z}^{\gamma} z}, \\
\left|v_{z}^{I}(z)\right\rangle=\left|k_{x}^{0}\right\rangle e^{i k_{z}^{0} z}-\sum_{\gamma=-\infty}^{\infty} R_{\gamma}\left|k_{x}^{\gamma}\right\rangle e^{-i k_{z}^{\gamma} z}
\end{gathered}
$$

Here the free space plane waves $\left\langle x \mid k_{x}^{\gamma}\right\rangle=\frac{e^{i k_{x}^{\gamma} x}}{\sqrt{\Lambda}}$, incident wave vector $\mathbf{k}_{0}=\left(k_{x}^{0}, k_{z}^{0}\right)$ and the scattered components $\mathbf{k}_{\gamma}$ $=\left(k_{x}^{\gamma},-k_{z}^{\gamma}\right)$ containing discrete diffraction order $\gamma$ in the range $\gamma=-\infty, \ldots, 0, \ldots, \infty$ comprising in-plane scattering where $k_{x}^{\gamma}=k_{x}^{0}+\frac{2 \pi}{L_{x}} \gamma$ and $k_{z}^{\gamma}=\sqrt{\left(n_{I} k_{0}\right)^{2}-\left(k_{x}^{\gamma}\right)^{2}}$ with $\frac{2 \pi}{L_{x}} \gamma$ representing the reciprocal-lattice vector in the primitive cell of constant $L_{x} . Y_{k}$ is the so-called admittance that governs the relationship between pressure and the velocity, derived from the momentum equation in Eq. (2) which for the reflected wave, in particular, is nothing but $Y_{k_{z}^{\gamma}}^{I}=c_{0} \rho_{p}^{I} \frac{k_{0}}{k_{z}^{\gamma}}$. Together with $\lambda=\frac{2 \pi}{k_{0}}$ we define the angle of incidence with respect to the normal of the surface as $\phi$, so with no loss of generality, $k_{z}^{\gamma}$ $=k_{0} \sqrt{n_{I}^{2}-\left(\sin \phi+\gamma \frac{\lambda}{L_{x}}\right)^{2}}$. If $\eta_{\gamma}=\sin \phi+\gamma \frac{\lambda}{L_{x}}$ we reach to the final simplifications of $k_{z}^{\gamma}=k_{0} \sqrt{n_{I}^{2}-\eta_{\gamma}^{2}}$ and henceforth $Y_{k_{z}^{\gamma}}^{I}$ $=\frac{c_{0} \rho_{p}^{I}}{\sqrt{n_{I}^{2}-\eta_{\gamma}^{2}}}$.

Region II $(0 \leq z \leq h)$ can be modeled as cavities with perfect rigid walls. Within this PRB approximation no sonic energy is penetrating into the material, which is valid for a broad range of frequencies, e.g., steel, brass, or concrete. Thus the boundary conditions in the apertures are as follows: $\frac{\partial p}{\partial \mathbf{n}}=0$ at $x= \pm \frac{a}{2}$, which is nothing but a vanishing normal component of the particle velocity $v_{x}$ with respect to the adjacent faces within the slit in a unit cell. This statement complies with $v_{z}$ to be zero at top and bottom interfaces corresponding to $z=0$ and $z=h$. The normalized modes of the cavity waveguide is given as $\langle x \mid \sigma, m\rangle=\sqrt{\frac{2-\delta_{0 m}}{a}} \cos q_{x}^{m}\left(x-x_{\sigma}\right.$ $\left.+\frac{a}{2}\right)$ if $\left|x-x_{\sigma}\right|<\frac{a}{2}$ otherwise zero, where $x_{\sigma}=\sigma \Lambda$ is the phase depicting the discrete groove $(\sigma)$ locations whereas the $m$ th slit waveguide mode is in the range $(m=0,1,2, \ldots, \infty)$ with $q_{x}^{m}=\frac{m \pi}{a}$. The entire eigenvalue expression for the field inside the slit is the following:

$$
\begin{gathered}
\left|p^{I I}(z)\right\rangle=\sum_{m, \sigma} Y_{q_{z}^{m}}^{I I}\left(A_{m, \sigma} e^{i q_{z}^{m} z}+B_{m, \sigma} e^{-i q_{z}^{m} z}\right)|m, \sigma\rangle, \\
\left|\mathrm{v}_{z}^{I I}(z)\right\rangle=\sum_{m, \sigma}\left(A_{m, \sigma} e^{i q_{z}^{m} z}-B_{m, \sigma} e^{-i q_{z}^{m} z}\right)|m, \sigma\rangle
\end{gathered}
$$

with wave vector and admittance as $q_{z}^{m}=\sqrt{\left(n_{I I} k_{0}\right)^{2}-\left(\frac{m \pi}{a}\right)^{2}}$ and $Y_{q_{z}^{m}}^{I I}=c_{0} \rho_{p}^{I I} \frac{k_{0}}{q_{z}^{m}}=\frac{c_{0} \rho_{p}^{I I}}{\sqrt{n_{I I}^{2}-\alpha_{m}^{2}}}$, respectively, while $\alpha_{m}=\frac{m \lambda}{2 a} . A_{m, \sigma}$ and $B_{m, \sigma}$ are the expanded wave amplitudes that are to be solved for in the matching procedure. Region (II), however, can further be decomposed according to the phase and groove cavities either on the side of wave irradiation or emission. If Eq. (4) describes the wave motion in the grooves at the side of wave irradiation, we express the groove modes in the emission side as

$$
\begin{gathered}
\left|p^{I I}(z)\right\rangle=\sum_{m, \sigma} Y_{q_{z}^{m}}^{I I}\left(C_{m, \sigma} e^{i q_{z}^{m}(z-h)}+D_{m, \sigma} e^{-i q_{z}^{m}(z-h)}\right)|m, \sigma\rangle, \\
\left|\mathrm{v}_{z}^{I I}(z)\right\rangle=\sum_{m, \sigma}\left(C_{m, \sigma} e^{i q_{z}^{m}(z-h)}-D_{m, \sigma} e^{-i q_{z}^{m}(z-h)}\right)|m, \sigma\rangle,
\end{gathered}
$$

whereas the only location where sound can propagate through the entire structure is within the central slit, which is when $\sigma=0$, 


$$
\begin{gathered}
\left|p^{I I}(z)\right\rangle=\sum_{m, 0} Y_{q_{z}^{m}}^{I I}\left(A_{m, 0} e^{i q_{z}^{m} z}+B_{m, 0} e^{-i q_{z}^{m} z}\right)|m, 0\rangle, \\
\left|\mathrm{v}_{z}^{I I}(z)\right\rangle=\sum_{m, 0}\left(A_{m, 0} e^{i q_{z}^{m} z}-B_{m, 0} e^{-i q_{z}^{m} z}\right)|m, 0\rangle .
\end{gathered}
$$

In the lowest region (III) $(z>h)$, the acoustic waves emerge in which the fields, as in Eq. (3), are expanded out in linear diffracted Bloch waves with $T_{\gamma}$ being the transmission coefficient,

$$
\begin{gathered}
\left|p^{I I I}(z)\right\rangle=\sum_{\gamma=-\infty}^{\infty} Y_{k_{z}^{\gamma}}^{I I I} T_{\gamma}\left|k_{x}^{\gamma}\right\rangle e^{i k_{z}^{\gamma}(z-h)}, \\
\left|v_{z}^{I I I}(z)\right\rangle=\sum_{\gamma=-\infty}^{\infty} T_{\gamma}\left|k_{x}^{\gamma}\right\rangle e^{i k_{z}^{\gamma}(z-h)} .
\end{gathered}
$$

Clearly Eq. (7) is a solution for waves traveling only in one direction, toward increasing values for $z$. At all interfaces the matching conditions are applied on the pressure $p$ while being projected over cavity modes $\left\langle m^{\prime}, \sigma^{\prime}\right|$ of indentation $\sigma^{\prime}$, such as on the fluid particle velocity $v_{z}$ that is projected over plane waves $\left\langle k_{x}^{\gamma^{\prime}}\right|$. It follows from this, that all linearexpansion coefficients in Eqs. (3)-(7) can be extracted, when one first imposes continuity in pressure $p$ at the openings and the endings of the slit/grooves, and second does so regarding the velocity $v_{z}$ though along the entire unitcell at the interfaces $z=0, h$. Following this sequential scheme, one creates a systematic top-down (from region I to III) wave mode coupling approach that with given attributes (functions), owns the ability to describe the entire acoustical problem under study, and yields a quantitative field representation through out all space. As we are mainly interested in subwavelength apertures, we consider that only the fundamental propagating slit eigenmode $(\langle x \mid \sigma, 0\rangle \rightarrow\langle x \mid \sigma\rangle)$ is excited. In this respect, we find it convenient to define some modal velocity fields with respect to the matched interfaces and the corresponding phase. For $\sigma \neq 0$ at the grooves: at the bottom of the grooves at the impinging side, the velocity $v_{z}^{I}\left(z=h_{g}=h_{i n}\right)$ vanishes due to the perfect rigid wall that the pressure field encounters, hence with $\psi_{i n}=e^{2 i k_{0} h_{i n}}$ the wave amplitudes read

$$
B_{\sigma}=\psi_{i n} A_{\sigma} .
$$

This statement is very useful in order to write down the identities for the modal field at the input side,

$$
\begin{gathered}
v_{\sigma}=A_{\sigma}-B_{\sigma}, \\
\epsilon_{i n} v_{\sigma}=A_{\sigma}+B_{\sigma} .
\end{gathered}
$$

Similar we can unravel expressions for the modal velocity at the emerging side with $\psi_{\text {out }}=e^{-2 i k_{0} h_{\text {out }}}$ inside the grooves $\left(h_{g}=h_{\text {out }}\right)$,

$$
D_{\sigma}=\psi_{\text {out }} C_{\sigma} .
$$

From this we also deduce the definitions for the modal velocity field at the output side,

$$
\begin{gathered}
v_{\sigma}^{\prime}=C_{\sigma}-D_{\sigma}, \\
\epsilon_{\text {out }} v_{\sigma}^{\prime}=C_{\sigma}+D_{\sigma} .
\end{gathered}
$$

$\epsilon_{\text {in }}$ and $\epsilon_{\text {out }}$ are equivalent to the bouncing back and forth of acoustic wave motion inside grooves placed either at the input or output side, respectively, as described, e.g., in Ref. 8. For $\sigma=0$ at the slit: the central slit is reminiscent to the conventional case where we impose continuity through an aperture. At that spatial location we match the open groove cavity with the slit waveguide modes and together with the definitions,

$$
\begin{aligned}
& v_{0}=A_{0}-B_{0}, \\
& v_{0}^{\prime}=D_{0}-C_{0},
\end{aligned}
$$

we hereby illustrate that the central slit, unlike for $\sigma \neq 0$, couples the incident to the emerging field that is occurring via the interface coupling function $G^{V}$,

$$
\begin{aligned}
& A_{0}+B_{0}=\epsilon_{0} v_{0}+G^{V} v_{0}^{\prime}, \\
& D_{0}-C_{0}=\epsilon_{0} v_{0}^{\prime}+G^{V} v_{0} .
\end{aligned}
$$

With all those terms defined in Eqs. (9)-(13) we can conclude the modal expansion by gathering all terms into the following system:

$$
\begin{aligned}
& \left(G_{\sigma \sigma}-\epsilon_{i n}\right) v_{\sigma}+\sum_{\sigma^{\prime} \neq \sigma} G_{\sigma \sigma^{\prime}} v_{\sigma^{\prime}}-\delta_{\sigma 0} G^{V} v_{0}^{\prime}=I_{\sigma}^{0}, \\
& \left(G_{\sigma \sigma}-\epsilon_{\text {out }}\right) v_{\sigma}^{\prime}+\sum_{\sigma^{\prime} \neq \sigma} G_{\sigma \sigma^{\prime}} v_{\sigma^{\prime}}^{\prime}-\delta_{\sigma 0} G^{V} v_{0}=0
\end{aligned}
$$

with

$$
\begin{gathered}
G^{V}=\frac{Y_{q_{z}^{0}}^{I I}}{\sin q_{z}^{0} h}, \\
\epsilon_{\text {in }}=\frac{Y_{q_{z}^{0}}^{I I}}{\tan q_{z}^{0} h_{\text {in }}}, \\
\epsilon_{\text {out }}=\frac{Y_{q_{z}^{0}}^{I I}}{\tan q_{z}^{0} h_{\text {out }}}, \\
\epsilon_{0}=\frac{Y_{q_{z}^{0}}^{I I}}{\tan q_{z}^{0} h} .
\end{gathered}
$$

The irradiation term $I_{\sigma}^{0}$ and the overlap function $G_{\sigma \sigma^{\prime}}$ are containing a phase with regards to indentation $\sigma^{\prime}$, as those terms govern the coupling to an incident wave and diffraction, respectively. The irradiation term reads

$$
I_{\sigma}^{0}=2 i Y_{k_{z}^{0}}^{I} S_{00}
$$

with $S_{00}=\sqrt{\frac{a}{\Lambda}} \operatorname{sinc} \frac{k_{x}^{0} a}{2} e^{-i k_{x}^{0} x_{\sigma}}$. The overlap function can be restated into a Green's function on a position basis, 


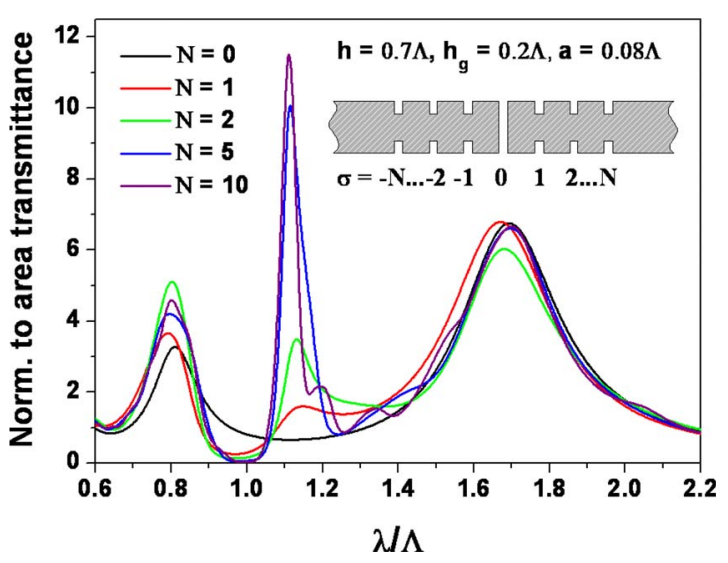

FIG. 2. (Color online) Influence on the normalized to area transmittance spectra, dependent on the numbers of surface corrugations ( $N$, see color legend for the value) with the geometries as specified in this figure. The acoustic plane wave is impinging normal incident on the structured system. The corrugations are symmetrically placed at the upper and lower side of the structure as the inset depicts.

$$
\begin{aligned}
G_{\sigma \sigma^{\prime}} & \rightarrow G_{x, x^{\prime}} \\
& =\left\langle x|\widetilde{G}| x^{\prime}\right\rangle \\
& =\frac{i \pi}{\lambda} \int_{x_{\sigma^{-}}-a / 2}^{x_{\sigma^{+}+a / 2}} \int_{x_{\sigma^{\prime}-a / 2}}^{x_{\sigma^{\prime}}+a / 2} H_{0}^{(1)}\left(k_{0}\left|x-x^{\prime}\right|\right) d x d x^{\prime}
\end{aligned}
$$

and be solved seminumerically, with $H_{0}^{(1)}$ the zero-order Hankel function of the first kind. The overlap function couples the fundamental $(m=0)$ groove $(\sigma \neq 0)$ or slit $(\sigma$ $=0)$ cavity mode inside indentation $\sigma$ over diffracted waves into indentation $\sigma^{\prime}$. Note in here how we have eliminated the supercell (discrete diffraction order) formalism into a continuous spectrum $L_{x} \rightarrow \infty$ representation.

\section{ANALYSIS}

In the following we shall acquire insight into the transmission properties of the single isolated slit milled into a sound-hard plate, Fig. 1, and deduce the importance of the surface corrugations. Concerning this matter, we will assume that the surrounding fluid is the same as inside the slit. In order to study the transmission efficiency, it is convenient to normalize the transmittance to the acoustic intensity that impinges on the slit, which is the following expression $T_{\text {slit }}$ $=\frac{1}{a} \operatorname{Im}\left(\sum_{\sigma} \delta_{\sigma 0} G^{V} v_{\sigma} v_{\sigma}^{\prime *}\right)$. We consider subwavelength slits and corrugations $(k a \ll 1)$ which justifies the assumption that only the fundamental waveguide mode is excited. Figure 2 shows the transmittance of a structure with $h=0.7 \Lambda, h_{g}=0.2 \Lambda$, and $a=0.08 \Lambda$ for various numbers of surface indentations $(N$ $=0,1,2,5,10)$, patterned both at the wave irradiation and emission side of the plate. The most apparent features in the transmittance spectra are two wide-banded peaks located at $\lambda \approx 0.8 \Lambda$ and $\lambda \approx 1.7 \Lambda$. These resonances that do not seem to be affected by the number of surface corrugations are determined by the overall plate thickness $h$, which governs the formation of standing waves in the slit. In other words, these peaks are associated to the excitation of slit-cavity modes of

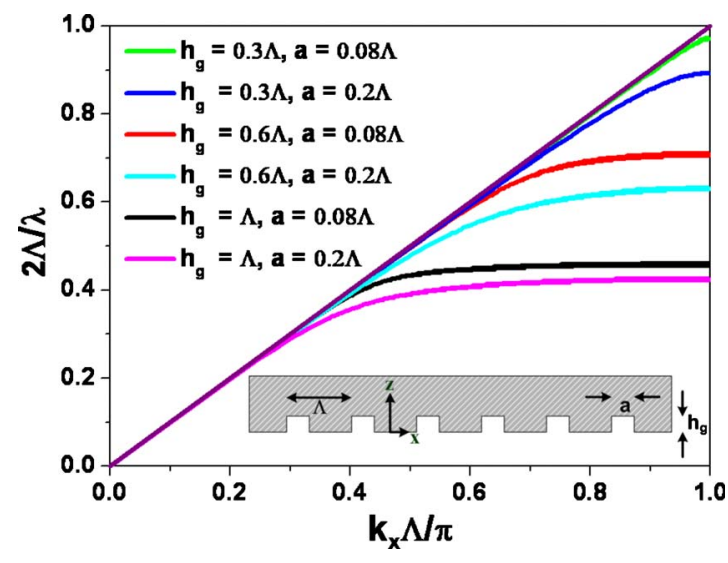

FIG. 3. (Color online) Inset shows a schematic representation of a groove grating where sound is incident in the positive $z$ direction. Bound modes dispersion relation, Eq. (21), for different geometrical parameter $a, h$ normalized to the period $\Lambda$ inside the first Brillouin zone. In here the influence of the groove depth and width on the band shape is calculated.

the FP type, which occurs at $\lambda_{\mathrm{FP}}=\frac{2 h}{l}$ for $l=1,2$ in this case. However, when the surface of the plate which is facing the sound irradiation side is patterned, we are able to excite bound surface modes which is giving rise to a huge enhancement of sound funneled through the subwavelength slit. Obviously those bound modes (BMs) must be leaky to ensure the coupling to radiating waves in the event of sound transmission. We will later elucidate the importance of the surface corrugations at the output side with regards to the sound emission. At a wavelength equal the period $\Lambda$, one finds a transmittance minimum for all cases $(\sigma \neq 0)$ which is the Woods anomaly. This minima becomes more apparent the higher the number of indentations is chosen, which is reminiscent to a groove grating when diffracted waves become grazing. This minimum is accompanied with a strong peak arising slightly above the period $(\lambda \gtrsim \Lambda)$, which is connected to the excitation of ASWs due to the periodicity of the structure, allowing incident sound binding to surface states. In order to shed some light on the above given transmittance properties, let us briefly recall the physical mechanisms involved when sound is impinging on a groove grating. ${ }^{19}$ This problem is easily described if we employ periodicity in our formalism and seek bound modes. The formalism, gathered with Eq. (14) can straightforwardly be applied to groove gratings by rejecting the homogeneous term as of no wave emerging interface and canceling the term responsible for the external irradiation $I^{0}$ as only bound surface states are sought, such as $G^{V}$ that cannot provide interface coupling to the output side due to the absence of perforations,

$$
G-\epsilon=0,
$$

now with $G=\frac{a}{\Lambda} \sum_{\gamma} \frac{k_{0}}{k_{\underline{\gamma}}^{\gamma}} \operatorname{sinc}^{2} \frac{k_{x}^{\gamma} a}{2}$. From Fig. 3 it becomes evident how the surface modes supported by a groove grating easily can be tuned by the groove width $a$ and depth $h_{g}$, controlling the bands location in the nearest proximity to the sound line and the flatness, respectively. For $\lambda>\Lambda \gg a$, when diffraction effects safely can be neglected, the overlap function reduces 


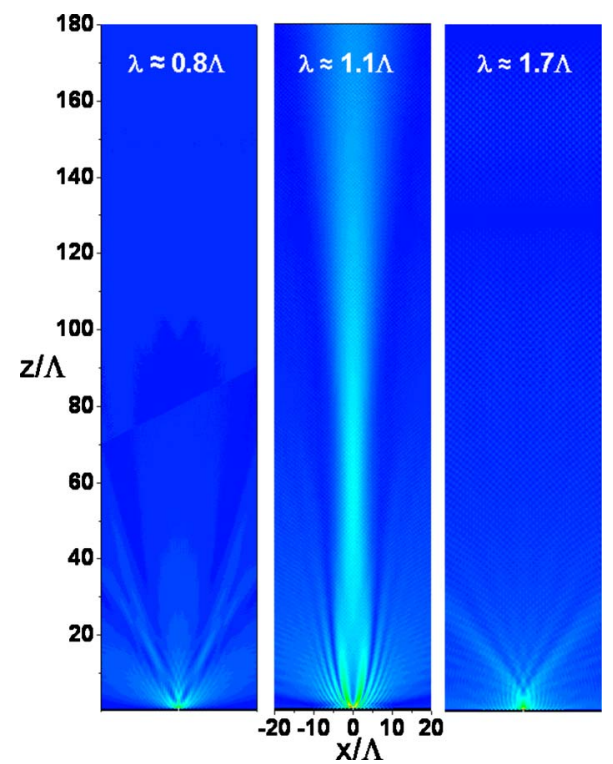

FIG. 4. (Color online) Pressure field $|p(x, z)|$ map in the far field for a structure containing $N=10$ surface corrugations symmetrically around the central slit and geometries as in Fig. 2. Each panel, as indicated from left to right corresponds to wavelengths $\lambda \approx 0.8 \Lambda$, $1.1 \Lambda$, and $1.7 \Lambda$, respectively. All field plots have been calculated with the mode matching technique described in the previous section.

to $G=\frac{a}{\Lambda} \frac{k_{0}}{\sqrt{k_{x}^{2}-k_{0}^{2}}}$ and consequently simplifies Eq. (21) into

$$
k_{x}=k_{0} \sqrt{1+\frac{a^{2}}{\Lambda^{2}} \tan ^{2} k_{0} h_{g}} .
$$

Equation (22) is an analytical dispersion relation of the addressed hybrid nature between groove-cavity modes and ASWs in a groove grating. This given, we can summarize the resonant mechanisms involved in the transmission process through the slit supported by the corrugations in the following context: Groove cavity modes $\lambda \approx 4 h_{g}$ : in order to boost sound through the central slit $(\sigma=0)$ mediated by the surface corrugations, obviously $v_{0}$ in Eq. (14) must be large, which is provided with large $v_{\sigma}$. For large values of $v_{\sigma}$ we can write: $\left(G_{\sigma \sigma}-\epsilon_{i n}\right) \approx 0$ which in the long-wavelength limit $\lambda$ $\gg a$ is determined by $\lambda=\frac{4 h_{g}}{2 n+1}$, where $n$ is an integer. Those cavity modes can also be determined from Eq. (22) in which $k_{x} \rightarrow \infty$. Interestingly, those bound modes resemble SPPs in a flat metal surface approaching $\omega_{s}=\omega_{p} / \sqrt{2}$, where $\omega_{p}$ is the plasma frequency of the metal. The asymptotic value in Eq. (22) is approached for infinite parallel momentum that yields an acoustic BM frequency for a groove grating: $\omega_{\mathrm{BM}}$ $=\pi c / 2 h_{g}$, here controlled by the groove height $h_{g}$. Slit waveguide modes $\lambda \approx 2 h$ : this component is governed by the thickness of the structure which is the excitation of FP resonances in the isolated slit. For $h_{g} \rightarrow 0$ in Eq. (14) we obtain the following resonant condition:

$$
\tan k_{0} h=\frac{2 \operatorname{Re}\left(G_{\text {slit }}\right)}{\left|G_{\text {slit }}\right|^{2}-1}
$$

that in the limit of extremely small apertures $\left(G_{\text {slit }} \rightarrow 0\right)$ predicts the appearance of transmission peaks close to the con-

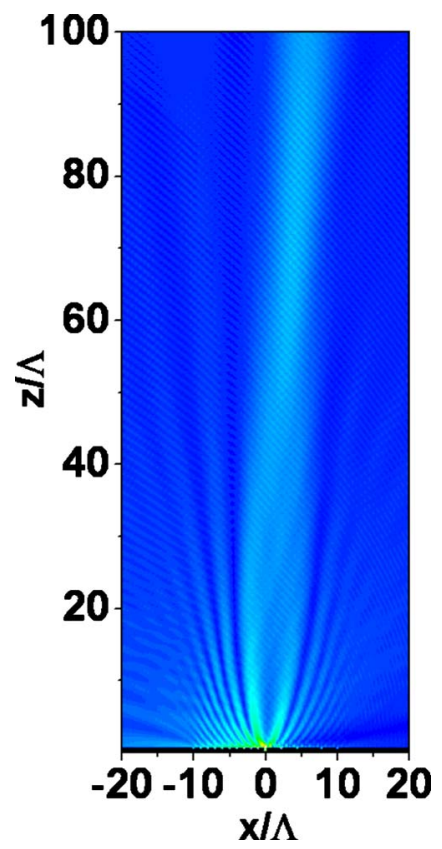

FIG. 5. (Color online) Beam steered pressure field $|p(x, z)|$ mapping of sound through a structure with same geometry as in Fig. 2 and $N=10$, though with unequal groove distances $\Lambda(\sigma>0)$ $=1.11 \Lambda(\sigma<0)$.

dition $\sin k_{0} h=0$, which are the peaks around $\lambda \approx 0.8 \Lambda$ and $\lambda \approx 1.7 \Lambda$ in Fig. 2 . Note also how this transmittance resonance, analytically is predictable by: $T_{\text {res }}^{\text {slit }}=\frac{\lambda}{\pi a} .{ }^{8}$ We have seen that the introduction of indentations does not affect these FP modes significantly. In-phase radiation $\lambda \approx \Lambda$ : this is the aforesaid resonance attributed to the excitation of surface modes of wavelength close to the period. At that particular wavelength, all sound emitted from the groove $\sigma$ over groove $\sigma^{\prime}$ reaches the central slit. This is the event leading to the emergence of a strong resonance $(\lambda \gtrsim \Lambda)$ seen in Fig. 2. In that particular figure, we see how all the three different mechanisms is giving rise to transmittance peaks, which further can be optimized by the right interplay of those components as adopted in Ref. 7 .

Apart from the ability to enhance the sound transmission through one subwavelength slit, we will now demonstrate how the diffraction of sound, when it emerges from a subwavelength aperture, can be controlled. This is accomplished with the corrugations at the emission side of the plate shown in Fig. 1, as they are affecting the formation of the beam when the acoustic wave is emerging the exit side of the structure. For a structure consisting of $N=10$ grooves and the geometries as captured in Fig. 2, we are calculating pressure field $|p(x, z)|$ maps in the far field. By means of an angular intensity distribution analysis (which we have not shown here), it is found that the used geometries is giving rise to a collimated beam of low angular divergence, where the farfield intensity strongly is influenced by $h_{g}$. All field plots in Fig. 4 clearly illustrate the presence of an enhanced acoustical transmission by virtue of a strong pressure-field confinement at the slit toward the output side of the structure. The pressure-field maps correspond to the transmittance peaks at $\lambda \approx 0.8 \Lambda, 1.1 \Lambda$, and $1.7 \Lambda$ illustrated in Fig. 2 . At $\lambda \approx 1.1 \Lambda$ 
though, an ASW is also excited at the output side of the structure, this then is scattered away by the grooves, and interferes constructively with the wave at the slit, giving rise to elongated focal spot in the far field, as has been illustrated in the central panel of Fig. 4. If now, for example, the unit length $\Lambda$ is chosen to be $10 \mathrm{~mm}$ in size and the sample is immersed in water, collimation of ultrasound aided by the excitation of ASWs is performed around 1.35 MHz. In order to perform elongated beam steering in the far field, a simple aperiodicity is introduced in the groove spacings. Consider a corrugated plate, with the geometries as depicted in Fig. 2. The discrete groove locations, as specified formerly, are $\sigma$ $>0$ for grooves located to the right-hand side (RHS) of the central slit $(\sigma=0)$ and $\sigma<0$ representing the left-hand side (LHS), all placed at the structures upper and lower side, simultaneously. The main difference in the geometries compared to the one in Fig. 2 is the period: $\Lambda(\sigma<0)=\Lambda(\sigma=0)$ and $\Lambda(\sigma>0)=1.11 \Lambda(\sigma<0)$. As a consequence of this anisotropic surface pattering, the collimated beam is inclined to the side of larger period $\Lambda(\sigma>0)=1.11 \Lambda(\sigma<0)$, as one may observe in Fig. 5 , for $\lambda \approx 1.15 \Lambda$.

\section{CONCLUSION}

For a single slit surrounded by corrugations within a perfect rigid plate, we have demonstrated how acoustic waves efficiently can be transferred through this aperture. It has been shown that the main mechanism giving rise to an enhanced transmission is governed by the right choice of geometrical parameters, which allows the coupling to FabryPerot, groove-cavity, and acoustic surface resonances. In particular, by flanking the transmission with surface corrugations, sound which is emerging the structure can be channeled in a well-defined direction as a collimated beam. By further scaling or tuning the structure, enhanced transmission such as the beaming effect can occur for various other frequencies. Moreover, it is also possible to control the spatial location of an elongated focal spot in an off-axis orientation, by texturing the surface corrugations with different periods at the LHS and the RHS of the central slit. Finally, this simple device for advance controlling of sound is an excellent candidate for ultrasonic detecting and scanning.

\section{ACKNOWLEDGMENTS}

This work was financially supported by the Spanish Ministry of Science and Innovation under Projects No. MAT2008-06609-C02 and No. CSD2007-046-Nanolight.es. J.C. gratefully acknowledges financial support from the Carlsberg Foundation under Contract No. QUANTONICS 2009-01-0167.

\footnotetext{
*Present address: Instituto de Optica, CSIC, Serrano 121, 28006 Madrid, Spain; johan.christensen@gmail.com

${ }^{1}$ T. W. Ebbesen, H. J. Lezec, H. F. Ghaemi, T. Thio, and P. A. Wolff, Nature (London) 391, 667 (1998).

${ }^{2}$ H. J. Lezec, A. Degiron, E. Deveux, R. A. Linke, L. MartinMoreno, F. J. Garcia-Vidal, and T. W. Ebbesen, Science 297, 820 (2002).

${ }^{3}$ J. B. Pendry, L. Martin-Moreno, and F. J. Garcia-Vidal, Science 305, 847 (2004).

${ }^{4}$ A. P. Hibbins, B. R. Evans, and J. R. Sambles, Science 308, 670 (2005).

${ }^{5}$ B. Hou, J. Mei, M. Ke, W. Wen, Z. Liu, J. Shi, and P. Sheng, Phys. Rev. B 76, 054303 (2007).

${ }^{6}$ M. H. Lu, X. K. Liu, L. Feng, J. Li, C. P. Huang, Y. F. Chen, Y. Y. Zhu, S. N. Zhu, and N. B. Ming, Phys. Rev. Lett. 99, 174301 (2007).

${ }^{7}$ J. Christensen, A. I. Fernandez-Dominguez, F. de Leon-Perez, L. Martin-Moreno, and F. J. Garcia-Vidal, Nat. Phys. 3, 851 (2007).

${ }^{8}$ J. Christensen, L. Martin-Moreno, and F. J. Garcia-Vidal, Phys. Rev. Lett. 101, 014301 (2008).

${ }^{9}$ J. Mei, B. Hou, M. Ke, S. Peng, H. Jia, Z. Liu, J. Shi, W. Wen,
}

and P. Sheng, Appl. Phys. Lett. 92, 124106 (2008).

${ }^{10}$ H. Estrada, P. Candelas, A. Uris, F. Belmar, F. Meseguer, and F. J. Garcia de Abajo, Appl. Phys. Lett. 93, 011907 (2008).

${ }^{11}$ H. Estrada, P. Candelas, A. Uris, F. Belmar, F. J. Garcia de Abajo, and F. Meseguer, Phys. Rev. Lett. 101, 084302 (2008).

${ }^{12}$ J. Christensen, P. A. Huidobro, L. Martin-Moreno, and F. J. Garcia-Vidal, Appl. Phys. Lett. 93, 083502 (2008).

${ }^{13}$ L. Martín-Moreno, F. J. García-Vidal, H. J. Lezec, A. Degiron, and T. W. Ebbesen, Phys. Rev. Lett. 90, 167401 (2003).

${ }^{14}$ F. J. García-Vidal, H. J. Lezec, T. W. Ebbesen, and L. MartínMoreno, Phys. Rev. Lett. 90, 213901 (2003).

${ }^{15}$ J. Bravo-Abad, F. J. Garcia-Vidal, and L. Martin-Moreno, Phys. Rev. Lett. 93, 227401 (2004).

${ }^{16}$ F. J. Garcia-Vidal, L. Martin-Moreno, T. W. Ebbesen, and L. Kuipers, Rev. Mod. Phys. 82, 729 (2010).

${ }^{17}$ L. D. Landau and E. M. Lifshitz, Fluid Mechanics, 3rd ed. (Butterworth-Heinemann, Oxford, 2000), Vol. 6.

${ }^{18}$ M. Willatzen, J. Sound Vib. 247, 719 (2001).

${ }^{19}$ L. Kelders, J. F. Allard, and W. Lauriks, J. Acoust. Soc. Am. 103, 2730 (1998); L. Kelders, W. Lauriks, and J. F. Allard, ibid. 104, 882 (1998). 